\title{
Complete Chloroplast Genome Sequence of Sonchus brachyotus Helps to Elucidate Evolutionary Relationships with Related Species of Asteraceae
}

\author{
Caixiang Wang $\mathbb{D}$, Juanjuan Liu, Yue Su, Meili Li, Xiaoyu Xie, and Junji Su $\mathbb{D}$ \\ College of Life Science and Technology, Gansu Agricultural University, Lanzhou 730070, China \\ Correspondence should be addressed to Junji Su; sujunjicotton@126.com \\ Caixiang Wang and Juanjuan Liu contributed equally to this work.
}

Received 26 July 2021; Revised 7 November 2021; Accepted 8 November 2021; Published 1 December 2021

Academic Editor: Fengjie Sun

Copyright (c) 2021 Caixiang Wang et al. This is an open access article distributed under the Creative Commons Attribution License, which permits unrestricted use, distribution, and reproduction in any medium, provided the original work is properly cited.

\begin{abstract}
Sonchus brachyotus DC. possesses both edible and medicinal properties and is widely distributed throughout China. In this study, the complete cp genome of $S$. brachyotus was sequenced and assembled. The total length of the complete $S$. brachyotus cp genome was $151,977 \mathrm{bp}$, including an LSC region of $84,553 \mathrm{bp}$, SSC region of $18,138 \mathrm{bp}$, and IR region of $24,643 \mathrm{bp}$. Sequence analyses revealed that the cp genome encoded 132 genes, including 87 protein-coding genes, 37 tRNA genes, and 8 rRNA genes. The GC content was 37.6\%. One hundred mononucleotide microsatellites, 4 dinucleotide microsatellites, 67 trinucleotide microsatellites, 4 tetranucleotide microsatellites, and 1 long repeat were identified. The SSR frequency of the LSC region was significantly greater than that of the IR and SSC regions. In total, 175 SSRs and highly variable regions were recognized as potential cp markers. By analyzing the IR/LSC and IR/SSC boundaries, structural differences between S. brachyotus and 6 other species were detected. According to phylogenetic analyses, S. brachyotus was most closely related to S. arvensis and S. oleraceus. Overall, this study provides complete $\mathrm{cp}$ genome resources for $S$. brachyotus that will be beneficial for identifying potential molecular markers and evolutionary patterns of $S$. brachyotus and its closely related species.
\end{abstract}

\section{Introduction}

Sonchus L. is a genus of annual, biennial, or perennial herbaceous plants in the Asteraceae (Compositae) family. Currently, the Sonchus genus includes 95 species [1-3], and it is widely distributed throughout Europe, Asia, Africa, and Pacific Islands [2]. Only 8 of the 95 species are distributed throughout China: Sonchus arvensis, Sonchus asper, Sonchus brachyotus, Sonchus lingianus, Sonchus oleraceus, Sonchus palustris, Sonchus transcaspicus, and Sonchus uliginosus. Specifically, they are distributed in Northeast, Northwest, North, Central, and South China and other regions and grow on mountain grassy slopes, roadsides, and fields with very rich resources, according to the Flora of China [4].

In China, S. brachyotus can be used not only as food but also as medicine to treat diseases [5]. S. brachyotus contains many major and trace elements that are important for the health and metabolism of the human body and is often used in health products as an edible plant [6-8]. When $S$. brachyotus is used as a medicinal whole herb, it has the function of clearing heat, detoxification, cooling blood, and stopping bleeding, and it is often used in the treatment of diseases such as acute pharyngitis, acute dysentery, appendicitis, enteritis, and hemorrhoids [6,9]. A previous study showed that $S$. brachyotus has antimicrobial activities against several pathogenic microorganisms [8]. For example, an extract from S. brachyotus can induce the apoptosis of A549 cells and inhibit their growth and proliferation, indicating that $S$. brachyotus can potentially be used to prevent and restrain tumor growth [10]. Pan et al. [11] also showed that an extract from $S$. brachyotus could exhibit antimicrobial activity against Escherichia coli, Enterobacter cloacae, Klebsiella 
pneumoniae, Salmonella enterica, Staphylococcus aureus, and Micrococcus luteus; this is especially true in the case of Escherichia coli. In addition, functional antioxidant components of S. brachyotus, including caffeic acid, rutin, orientin, and luteolin, can scavenge free radicals [12]. Although the chemical composition of $S$. brachyotus has been reported, $S$. brachyotus and $S$. arvensis are similar in morphology and difficult to distinguish, and their phylogenetic relationships are not very clear.

The chloroplast is an important plastid that provides necessary energy for growth via photosynthesis and plays vital roles in the physiology and development of plants. Chloroplasts, as semiautonomous organelles, possess a genetic information expression system. In contrast to nuclear DNA, chloroplast (cp) DNA exhibits single-parent inheritance. The $\mathrm{cp}$ genome is more conserved than mitochondrial and nuclear genomes in terms of gene type, genome organization, and genome structure [13], so the $\mathrm{cp}$ genome has become an important means for reconstructing the phylogenetic relationships among plant species [14-21]. With the development of bioinformatics analysis and sequencing technology, studies on the evolution of species using cp genome sequences are increasing.

In this study, we sequenced and analyzed the complete cp genome of S. brachyotus and reconstructed the phylogeny of Compositae based on the cp genomes of 42 species. The following questions were addressed: (1) what are the features of the cp genome of S. brachyotus? (2) How many potential microsatellite markers can the $\mathrm{cp}$ genome provide? (3) Which types of structural variation events have occurred across the cp genomes in the Sonchus genus?

\section{Materials and Methods}

2.1. Preparation of Materials. The plant material was transplanted from the shore of the Yellow River in Anning District of Lanzhou, Gansu Province, China $\left(36^{\circ} 5^{\prime} 10^{\prime} \mathrm{N}, 103^{\circ} 34^{\prime} 47^{\prime} \mathrm{E}\right)$ to pots in a laboratory (Figure S1). Then, fresh leaf tissue (1-2 grams) was sent to Genepioneer Biotechnologies Inc., Nanjing, 210023, China, for sequencing.

2.2. DNA Extraction, Genome Sequencing, and Annotation. Total genomic DNA was extracted from $100 \mathrm{mg}$ of fresh leaves of $S$. brachyotus using the CTAB (cetrimonium bromide) method [22]. The Illumina NovaSeq 6000 platform was used to construct and sequence a genomic library on the basis of the standard Illumina paired-end (PE) protocol. The raw reads were trimmed using NGS QC Toolkit_v2.3.3 [23]. After trimming of low-quality reads and adapter sequences, the clean reads were aligned with the reference genome of Lactuca sativa (NC_007578.1) and S. arvensis (NC_054161) from the NCBI GenBank database using Burrows-Wheeler Alignment (BWA) [24], and sequenced reads of chloroplast genomes were "selected" from clean sequence data. The matched PE reads were assembled using SPAdes v3.10.1 software [25]. The reference sequences of the genomes were compared for collinearity of conserved and rearranged genomes by MUMmer v3.23 [26]. Annotation was performed with BLAST v2.2.25 (https://blast.ncbi.nlm
.nih.gov/Blast.cgi), HMMER v3.1b2 (http://http://www .HMMER.org/), and Aragorn v1.2.38 (http://130.235.244 92/ARAGORN/). BLAST v2.2.25 was applied to compare coding sequences (CDSs) of chloroplasts in the NCBI database, the preliminary draft annotation was examined and adjusted manually by comparison with the reference cp genome, and the gene annotation results of the cp genome were then obtained. The rRNA and tRNA annotation information was obtained by using HMMER v3.1b2 and Aragorn v1.2.38 to compare the rRNA and tRNA sequences of chloroplasts in the NCBI online database. The annotated cp DNA sequences were submitted to the NCBI database by BankIt to obtain the GenBank sequence login number MT850048. OGDRAW v1.1.1 software [27] was then used to map the cp genomes of $S$. brachyotus according to the chloroplast sequence assembly results.

\subsection{Repeat Structure and Sequence Analysis. Vmatch v2.3.0} (http://www.vmatch.de/) was utilized to explore the reduplicative structure of the cp genome of $S$. brachyotus and to locate a variety of styles of repeat sequences for forward, palindromic, inverted, and complementary sequences. The parameters were set to a minimum length of $30 \mathrm{bp}$ and a Hamming distance of 3. Microsatellite (mono-, di-, tri-, tetra-, penta-, and hexanucleotide repeats) detection was performed using MISA v1.0 (http://pgrc.ipk-gatersleben.de/misa/misa.html), with parameters of $1-8$ (mononucleotide motifs with a minimum of 8 repetitions), 2-5, 3-3, 4-3, 5-3, and 6-3.

2.4. Identification of Highly Divergent Regions. We used published $\mathrm{cp}$ genome sequences of 6 species of Sonchus, namely, Sonchus webbii (GenBank accession number NC_042383), Sonchus acaulis (NC_042382), Sonchus canariensis (NC_ 042381), Sonchus boulosii (NC_042244), Sonchus arvensis (NC_054161.1), and Sonchus oleraceus (MG878405), to analyze the borders and synteny of the inverted repeat (IR) and single-copy (SC) regions of S. brachyotus and the above 6 species. We used IRScope software (https://irScope .shinyapps.io/Irapp/) to generate a comparison diagram of the IR boundary [28]. Entire genome sequences were evaluated to appraise realignments and extensive sequence variances using Mauve 2.3.1 [29]. Moreover, the cp genome was arranged using MAFFT v7.427 [30] to identify divergence hotspots, after which sliding window analyses were conducted via DnaSP v5 [31] to determine the nucleotide diversity $(\mathrm{Pi})$ of the complete $\mathrm{cp}$.

2.5. Phylogenetic Analyses. A total of $43 \mathrm{cp}$ genomes available in GenBank were recovered to infer the phylogenetic relationships, including newly sequenced S. brachyotus and 42 published Compositae species (Table S1). Multiple alignments were performed using complete $\mathrm{cp}$ genomes based on the conserved structure and gene order of the chloroplast genomes. All the nucleotide sequences were aligned using MAFFT v7.308 [32] to assess the taxonomic and phylogenetic relationships of $S$. brachyotus. Two methods were employed to construct phylogenetic trees, including maximum parsimony (MP) and Bayesian inference (BI). MP analyses were performed using Mega 
TABLe 1: Summary of the features of 7 Sonchus chloroplast genomes.

\begin{tabular}{|c|c|c|c|c|c|c|c|c|c|}
\hline \multirow{2}{*}{ Taxon } & \multicolumn{4}{|c|}{ Length (bp) } & \multicolumn{4}{|c|}{ Number of genes } & \multirow{2}{*}{ GC content $(\%)$} \\
\hline & Genome & LSC & SSC & IR & Total & Protein coding & tRNA & rRNA & \\
\hline S. brachyotus & 151,977 & 84,553 & 18,138 & 24,643 & 132 & 87 & 37 & 8 & 37.6 \\
\hline S. arvensis & 151,967 & 84,251 & 18,184 & 24,766 & 130 & 87 & 37 & 6 & 37.6 \\
\hline S. oleraceus & 151,808 & 84,142 & 18,217 & 24,739 & 130 & 87 & 37 & 6 & 37.6 \\
\hline S. boulosii & 152,016 & 83,988 & 18,566 & 24,731 & 130 & 88 & 36 & 6 & 37.6 \\
\hline S. acaulis & 152,017 & 84,355 & 18,244 & 24,746 & 131 & 88 & 37 & 6 & 37.6 \\
\hline S. canariensis & 152,075 & 84,338 & 18,245 & 24,746 & 131 & 88 & 37 & 6 & 37.6 \\
\hline S. webbii & 152,194 & 84,269 & 18,409 & 24,758 & 131 & 88 & 37 & 6 & 37.6 \\
\hline
\end{tabular}

LSC: large single copy; SSC: small single copy; IR: inverted repeat; tRNA: transfer RNA; rRNA: ribosomal RNA.

11.0 software [33], and the addition sequence was set as 1,000 replications for the heuristic search. BI analyses were conducted using MrBayes v3.2.6 [34] based on the model GTR $+\mathrm{G}$ inferred from Modeltest 3.7 [35]. The first 25\% of trees generated were discarded as burn-in, and the remaining trees were used to construct a majority-rule consensus tree with posterior probability (PP) values for each node.

\section{Results}

3.1. Chloroplast Genome Features, Sequencing, and Assembly of $S$. brachyotus. After trimming of low-quality reads and adapter sequences, the total length of the reads was approximately $7.5 \mathrm{~Gb}$ and $24,858,121$ clean reads were produced by the Illumina NovaSeq 6000 platform. Based on a combination of de novo and reference-guided assembly, the cp genome of $S$. brachyotus was obtained. The complete cp genome sequence of $S$. brachyotus was submitted to the NCBI database under GenBank accession number MT850048. The total length of the cp genome of S. brachyotus was 151,977 bp (Table 1, Figure 1). The cp genome contained four characteristic regions: a large single-copy (LSC) region of $84,553 \mathrm{bp}$, a small single-copy (SSC) region of $18,138 \mathrm{bp}$, and a pair of inverted repeats (IRa and IRb) of $24,643 \mathrm{bp}$. The base composition of the complete cp genome sequence was analyzed and found to be $31.3 \% \mathrm{~T}, 31.1 \% \mathrm{~A}$, $18.7 \% \mathrm{C}$, and $18.9 \% \mathrm{G}$. The overall GC content was $37.6 \%$, which is very close to those of other Sonchus species. Furthermore, the GC contents were unevenly distributed across regions of the cp genome and were found to be $35.71 \%$, $31.44 \%$, and $43.08 \%$ for the LSC, SSC, and IR regions, respectively.

The $S$. brachyotus cp genome included 132 genes, 1 or 2 more genes than the other 6 Sonchus genomes, of which there were 87 protein-coding genes, 8 rRNA genes, and 37 tRNA genes (Table 1). Eight protein-coding genes ( $n d h B$, rpI2, rpI23, rps7, rps12, $y c f 2, y c f 15$, and $y c f 1), 7$ tRNA genes (trnI-CAU, trnL-CAA, trnV-GAC, trnI-GAU, trnA-UGC, trnR-ACG, and trnN-GUU), and 4 rRNA genes (rrn16, $r r n 23, r r n 4.5$, and $r r n 5)$ were duplicated in the IR region in the $\mathrm{cp}$ genomes. There were 113 unique genes, and 16 genes (trnK-UUU, rps16, rpoC1, atpF, trnG-UCC, trnL-UAA, trnV$U A C, r p s 12$, petB, petD, rpl16, rpl2, ndhB, trnI-GAU, trnA$U G C$, and $n d h A)$ contained 1 intron, whereas 2 protein- coding genes $(y c f 3$ and $c l p P)$ contained 2 introns (Table 2). The majority of these intron-containing genes were located in the LSC region.

3.2. Simple Sequence Repeats and Large Repeat Sequences. In this study, we explored the presence of various microsatellites (mono-, di-, tri-, tetra-, penta-, and hexanucleotides) in the cp genome of $S$. brachyotus. A total of 175 microsatellites were detected in the cp genome of S. brachyotus, and the most common simple sequence repeats (SSRs) were mononucleotides (notably for A/T), with 100, accounting for $57 \%$ of the SSRs in S. brachyotus. The second most abundant motif type was the trinucleotide type, especially TAA, with a total number of 67 in S. brachyotus (approximately 38\%). The proportion of other SSR types was relatively low (approximately 2\% for dinucleotides and tetranucleotides). Intriguingly, the SSRs in S. brachyotus were chiefly distributed in coding regions $(46.5 \%)$, with much lower numbers distributed in noncoding introns (12.6\%) and intergenic regions (41\%). The SSRs were spaced disproportionately through the cp genome, with the largest number of SSRs situated in the LSC region, followed by the IR and SSC regions, in the quadripartite structure regions (Figure 2(a)).

Repeat motifs are valuable for phylogenetic reconstruction. Consequently, we examined the forward, palindromic, complementary, and reverse repeats in the S. brachyotus cp genome (Figure 2(b)). Overall, 35 pairs of repeat sequences were identified in the cp genome of $S$. brachyotus, which contained 16 palindromic repeats and 19 forward repeats; however, complementary and reverse repeats were not found in S. brachyotus. The lengths of the repeats ranged from 30 to 24,643 bp in S. brachyotus, and the most common repeat length was $30 \mathrm{bp}$ (approximately 34\%), followed by repeats of $43 \mathrm{bp}(11 \%)$ and $31-42 \mathrm{bp}$ (approximately less than 10\%), while those of 43-24,643 bp (approximately $2 \%)$ were comparatively rare. The repeats were mainly distributed in noncoding regions, including intergenic spacers (IGSs) and introns. However, several coding and tRNA genes, such as $y c f 2, y c f 3, p s b N, p s a B, p s a A, n d h A, r p I 16$, and $t r n S$, also contained repeat sequences.

3.3. Expansion and Contraction of Border Regions. The expansion and contraction of the borders and adjacent genes of cp genomes give rise to genome size variations among various plant lineages. Hence, the borders and adjacent 

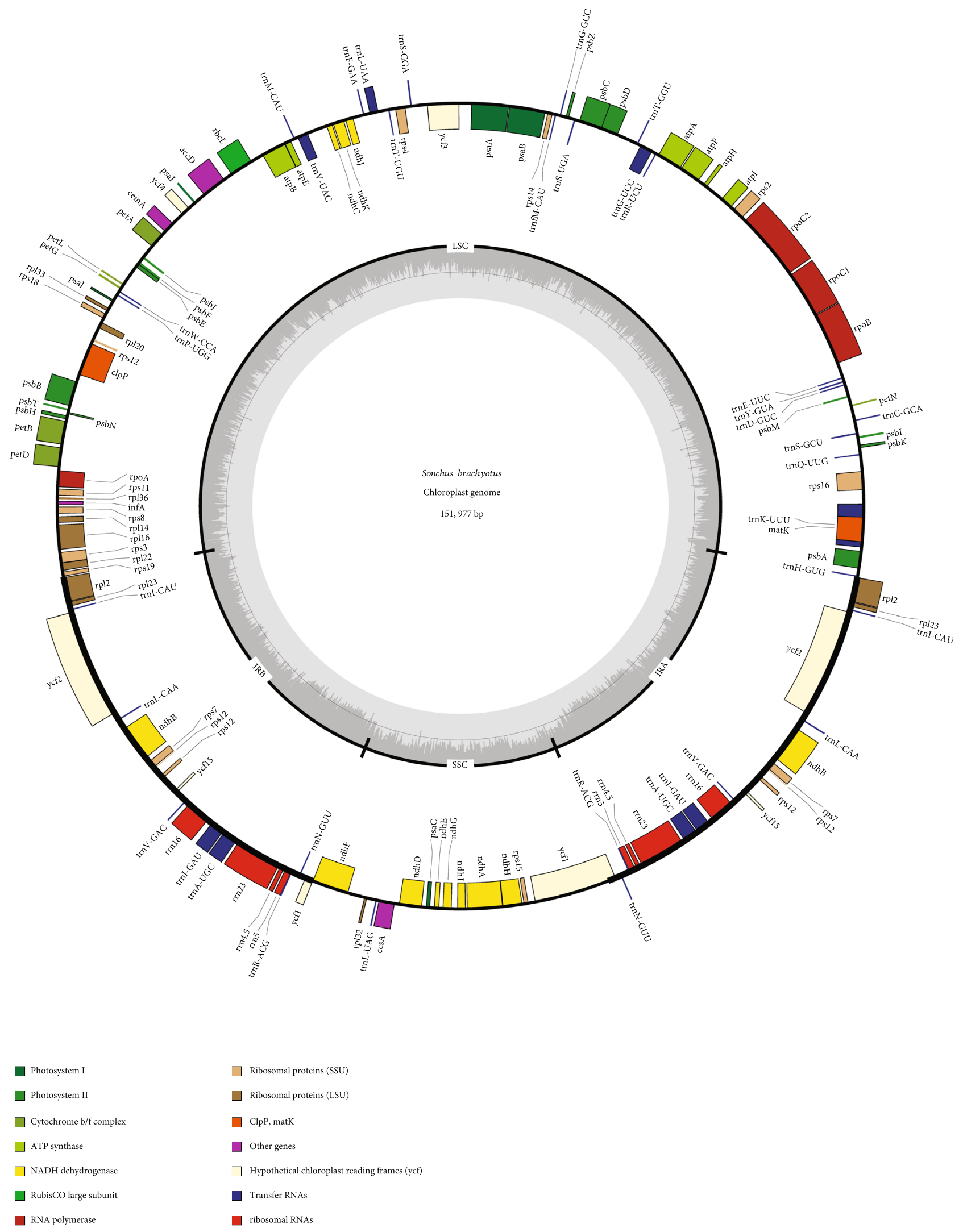

Figure 1: Gene map of the S. brachyotus chloroplast genome. The genes inside and outside of the circle are transcribed in the clockwise and counterclockwise directions, respectively. Genes belonging to different functional groups are indicated in different colors. The thick lines indicate the extent of the inverted repeats (IRa and IRb) that separate the genomes into small single-copy (SSC) and large single-copy (LSC) regions. 
TABLE 2: List of genes found in the chloroplast genome of S. brachyotus.

\begin{tabular}{|c|c|c|}
\hline $\begin{array}{l}\text { Category of } \\
\text { genes }\end{array}$ & Group of genes & Names of genes \\
\hline \multirow{5}{*}{$\begin{array}{l}\text { Self- } \\
\text { replication }\end{array}$} & $\begin{array}{l}\text { Large subunit of ribosome } \\
\text { (LSU) }\end{array}$ & rpl33, rpl20, rpl36, rpl14, $\operatorname{rpl} 16^{*}, \operatorname{rpl} 22, \operatorname{rpl} 2(2)^{*}, \operatorname{rpl} 23(2), \operatorname{rpl} 32$ \\
\hline & $\begin{array}{l}\text { Small subunit of ribosome } \\
\text { (SSU) }\end{array}$ & rps16 ${ }^{*}, \operatorname{rps} 2, \operatorname{rps} 14, \operatorname{rps} 4, \operatorname{rps} 18, \operatorname{rps} 12(2)^{*}, \operatorname{rps} 11, \operatorname{rps} 8, \operatorname{rps} 3, \operatorname{rps} 19, \operatorname{rps} 7(2), \operatorname{rps} 15$ \\
\hline & RNA polymerase subunits & rpoB, rpoC1* , rpoC2, rpoA \\
\hline & Ribosomal RNA genes & $\operatorname{rrn} 16(2), \operatorname{rrn} 23(2), \operatorname{rrn} 4.5(2), \operatorname{rrn} 5(2)$ \\
\hline & Transfer RNAs (tRNAs) & 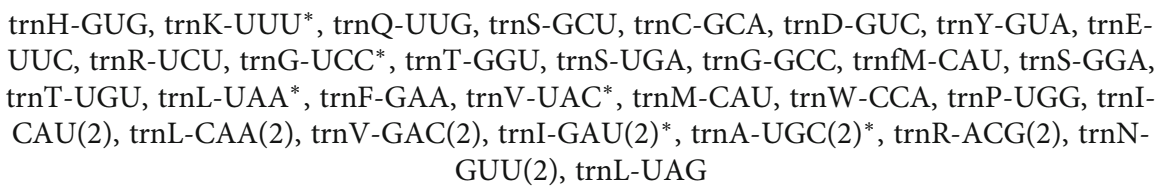 \\
\hline \multirow{6}{*}{ Photosynthesis } & Photosystem I & psaB, psaA, ycf3**, psaI, ycf4, psaJ, psaC \\
\hline & Photosystem II & psbA, psbK, psbI, psbM, psbD, psbC, psbZ, psbJ, psbF, psbE, psbB, psbT, psbN, psbH \\
\hline & $\begin{array}{l}\text { Subunits of NADH } \\
\text { dehydrogenase }\end{array}$ & ndhJ, ndhK, ndhC, ndhB $(2)^{*}$, ndhF, ndhD, ndhE, ndhG, ndhI, ndhA*, ndhH \\
\hline & Cytochrome b/f complex & petN, petA, petL, petG, petB*, petD* \\
\hline & ATP synthase & atpI, atpH, atpF*, atpA, atpE, atpB \\
\hline & Large chain of rubisco & rbcL \\
\hline \multirow{7}{*}{ Other genes } & $\begin{array}{l}\text { Translation initiation } \\
\text { factor }\end{array}$ & $\operatorname{infA}$ \\
\hline & Maturase & matK \\
\hline & Protease & $\operatorname{clpP} P^{* *}$ \\
\hline & $\begin{array}{l}\text { Envelope membrane } \\
\text { protein }\end{array}$ & cemA \\
\hline & $\begin{array}{l}\text { Subunit of acetyl-CoA- } \\
\text { carboxylase }\end{array}$ & $\mathrm{accD}$ \\
\hline & $\begin{array}{l}\text { Cytochrome } \mathrm{c} \text { biogenesis } \\
\text { protein }\end{array}$ & $\operatorname{ccs} \mathrm{A}$ \\
\hline & $\begin{array}{l}\text { Hypothetical chloroplast } \\
\text { reading frames }\end{array}$ & $y c f 2(2), y c f 15(2), y c f 1(2)$ \\
\hline
\end{tabular}

${ }^{*}$ Genes containing a single intron. ${ }^{* *}$ Genes containing 2 introns.

genes of the other 6 published Sonchus plant species were compared with those of $S$. brachyotus to analyze the expansion and contraction diversification in connection regions (Figure 3). The entire genome structure, the gene order, and the gene number were conserved, as were the IRb/SSC and IRa/LSC boundaries of the seven Sonchus cp genomes. The rps19 genes in the LSC region of the 6 species were amplified and generated products of 87 and $89 \mathrm{bp}$ (89 bp for S. oleraceus, S. boulosii, S. canariensis, S. acaulis, and S. arvensis; $87 \mathrm{bp}$ for $S$. webbii) for the IRb region; in S. brachyotus, this gene was completely situated in the LSC region, and the distance to the connection was $31 \mathrm{bp}$. The rpl2 gene in the IR regions was $27,145,146$, and $147 \mathrm{bp}$ from the LSC in the 7 species ( 27 bp for S. brachyotus; 145 bp for S. webbii; $146 \mathrm{bp}$ for S. boulosii; and $147 \mathrm{bp}$ for S. oleraceus, S. canariensis, S. acaulis, and $S$. arvensis). The $\operatorname{trn} H$ gene in the LSC region was contracted by $1,2,3$, and $32 \mathrm{bp}$ from the connection region of IRa/LSC ( 1 bp for S. webbii; 2 bp for S. brachyotus, S. boulosii, S. canariensis, and S. acaulis; $3 \mathrm{bp}$ for S. arvensis; and $32 \mathrm{bp}$ for $S$. oleraceus). The $y c f 1$ gene spanning the SSC/IRb junction showed a length of $44 \mathrm{bp}$ in S. oleraceus, S. boulosii, S. canariensis, S. acaulis, and $S$. arvensis, but in S. brachyotus and S. webbii, it showed a length of 11 and $2 \mathrm{bp}$. The $n d h F$ gene was located completely within the SSC region, and the distance to the IRb/SSC junction was 0,5 , and $14 \mathrm{bp}$. The $y c f 1$ gene extended over the boundary region between the SSC and IRa regions. The $t r n N$ gene was located entirely within IRa and was contracted by $793-814 \mathrm{bp}$. The variations in the IR/SC boundary regions in the 7 Sonchus $\mathrm{cp}$ genomes were responsible for the length differences in the four regions and whole genome sequences.

3.4. Sequence Divergence and Hot Spots. To clarify the level of genomic differences, the cp genome sequences of $S$. brachyotus plants were compared via Mauve. The local collinear block sequences (LCBSs) confirmed by Mauve showed high sequence similarity among the 7 Sonchus cp genomes, which indicated that the genome structure was quite conserved at the gene sequence level (Figure 4). As anticipated, the SC 


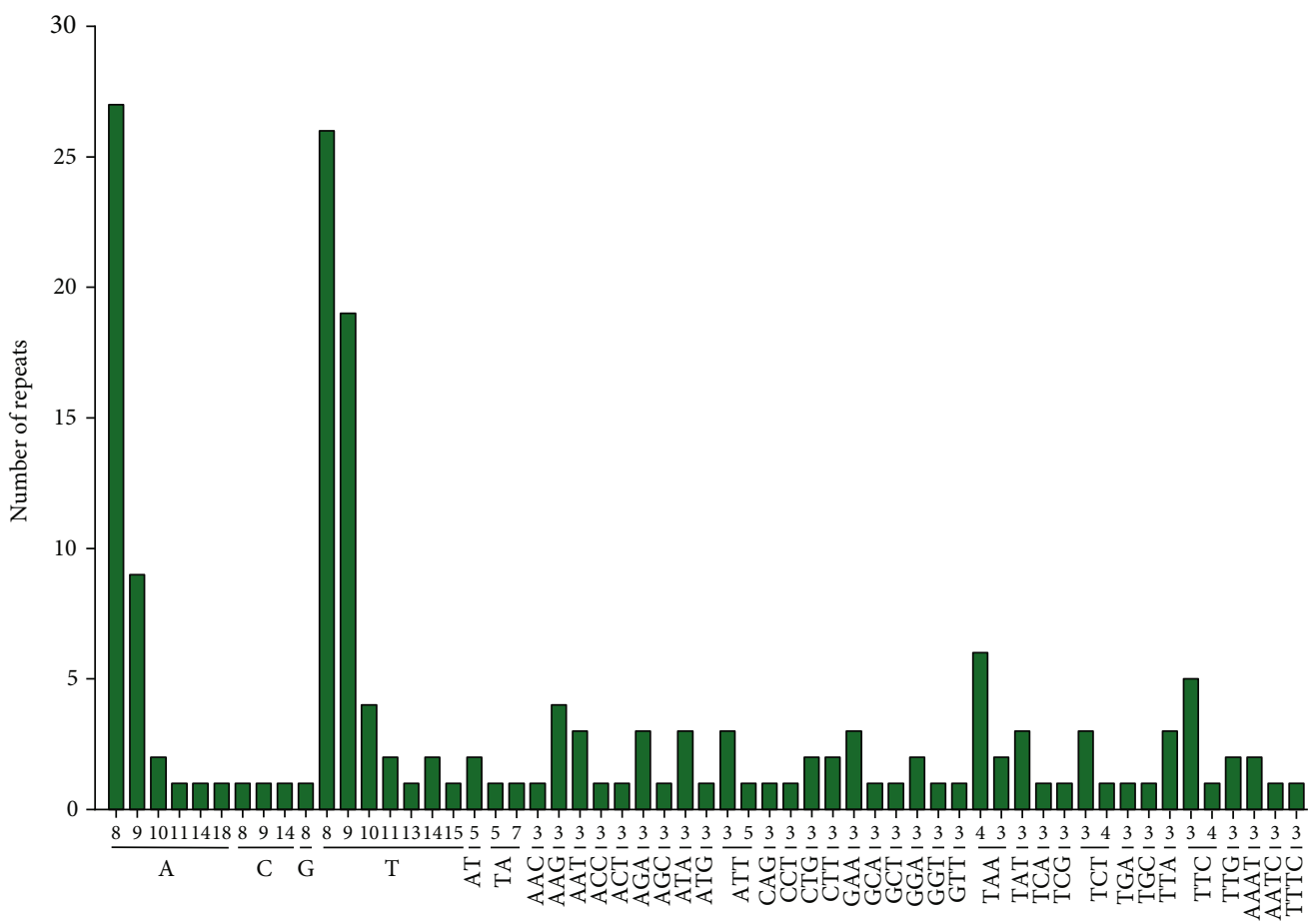

Length of repeat $(\mathrm{bp})$ and repeated sequences

(a)

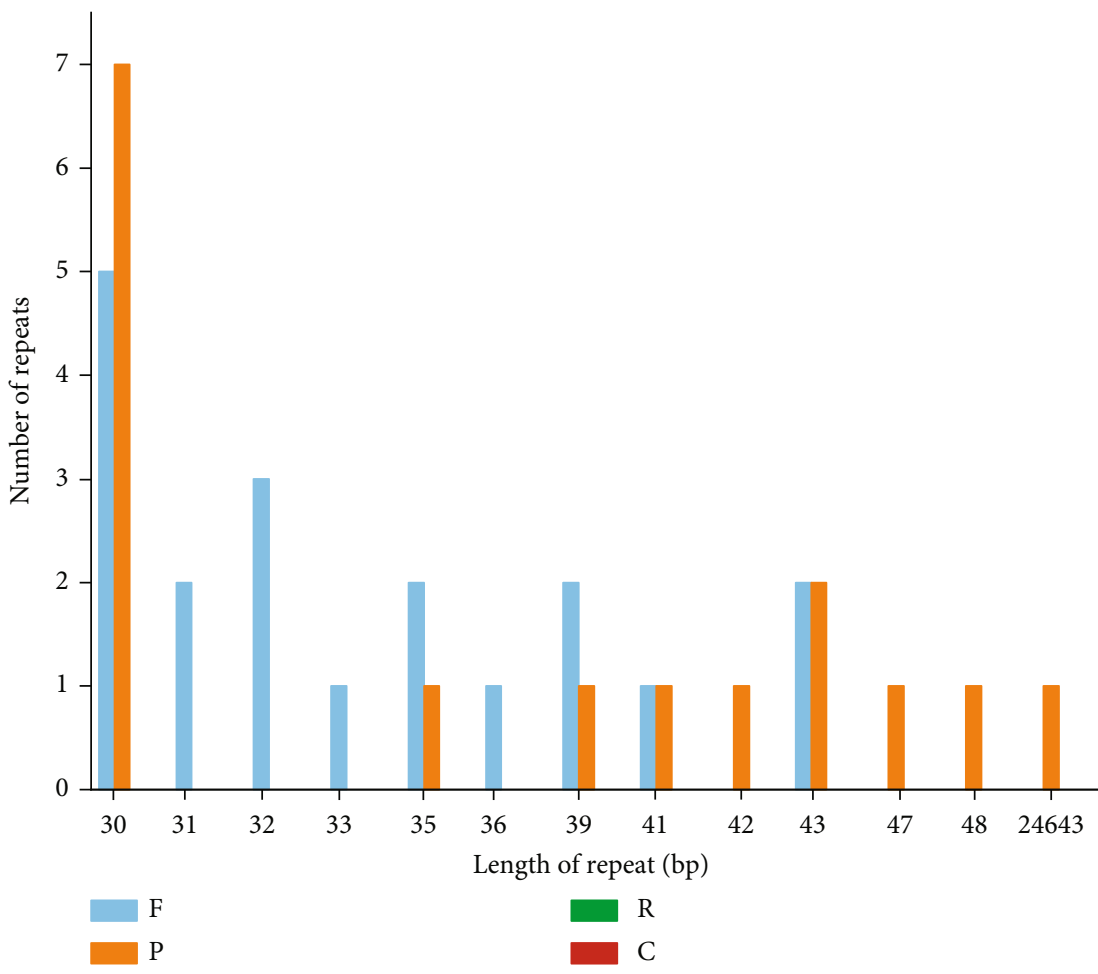

(b)

FIGURE 2: SSR numbers according to their distribution and repeat type and repeat numbers according to repeat type and repeat length in $S$. brachyotus. (a) Number of SSR motifs in S. brachyotus. (b) Variation in the distribution of forward (F), reverse (R), complementary (C), and palindromic $(\mathrm{P})$ repeats and the number of different repeats in the chloroplast genome of $S$. brachyotus.

regions were less conserved than the IR regions. The most divergent areas were 5,000-20,000, 25,000-40,000, 45,00080,000 , and $110,000-130,000$ bp in size.
We generated 113 loci from S. brachyotus and calculated the $\mathrm{Pi}$ value of each gene with VCFtools. The Pi values obtained from S. brachyotus ranged from 0 to 0.099 ( $y c f 1)$. 


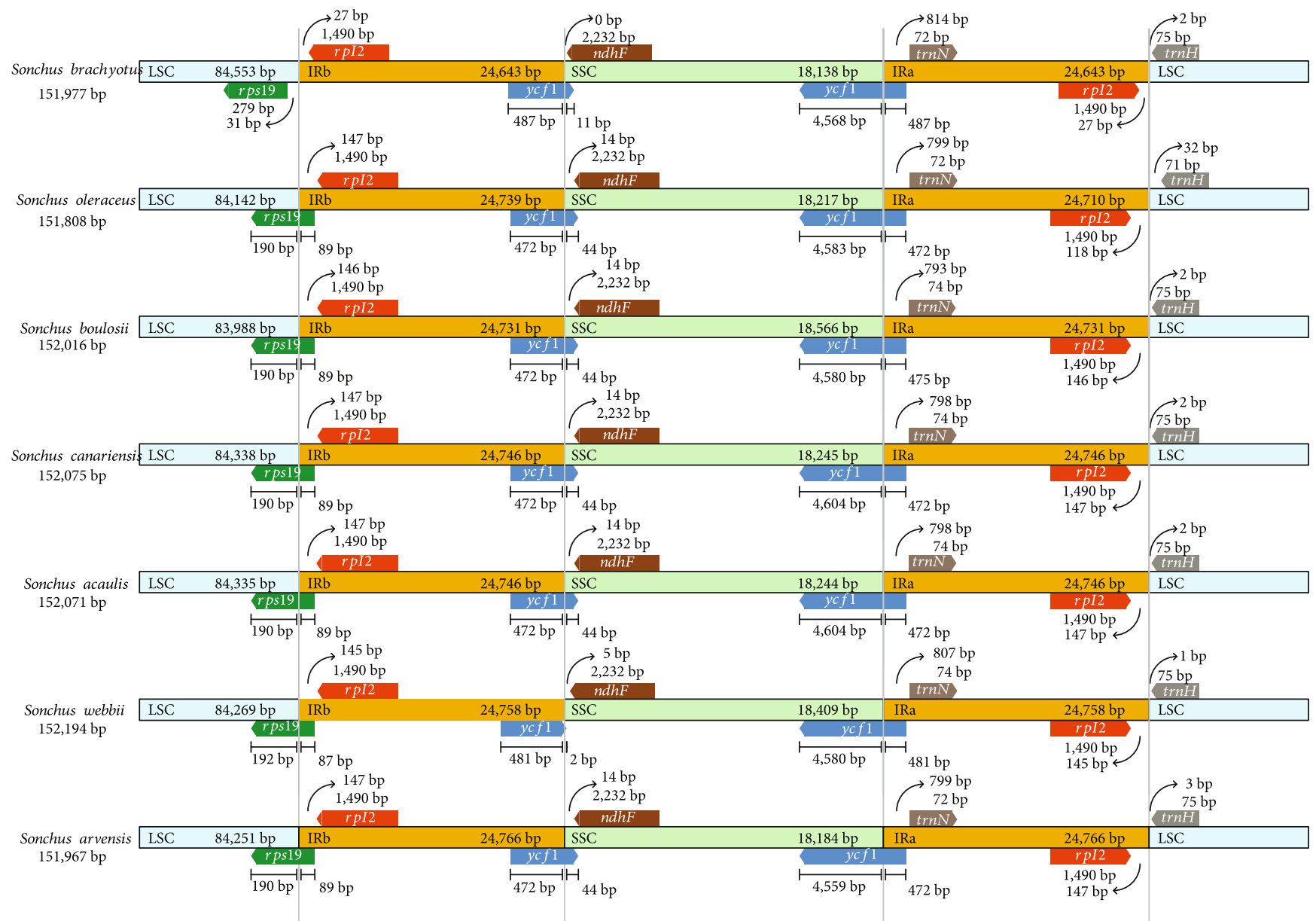

FIGURE 3: Comparison of the border positions of LSC, SSC, and IR regions among 7 chloroplast genomes from Sonchus species. Gene names are indicated in boxes.

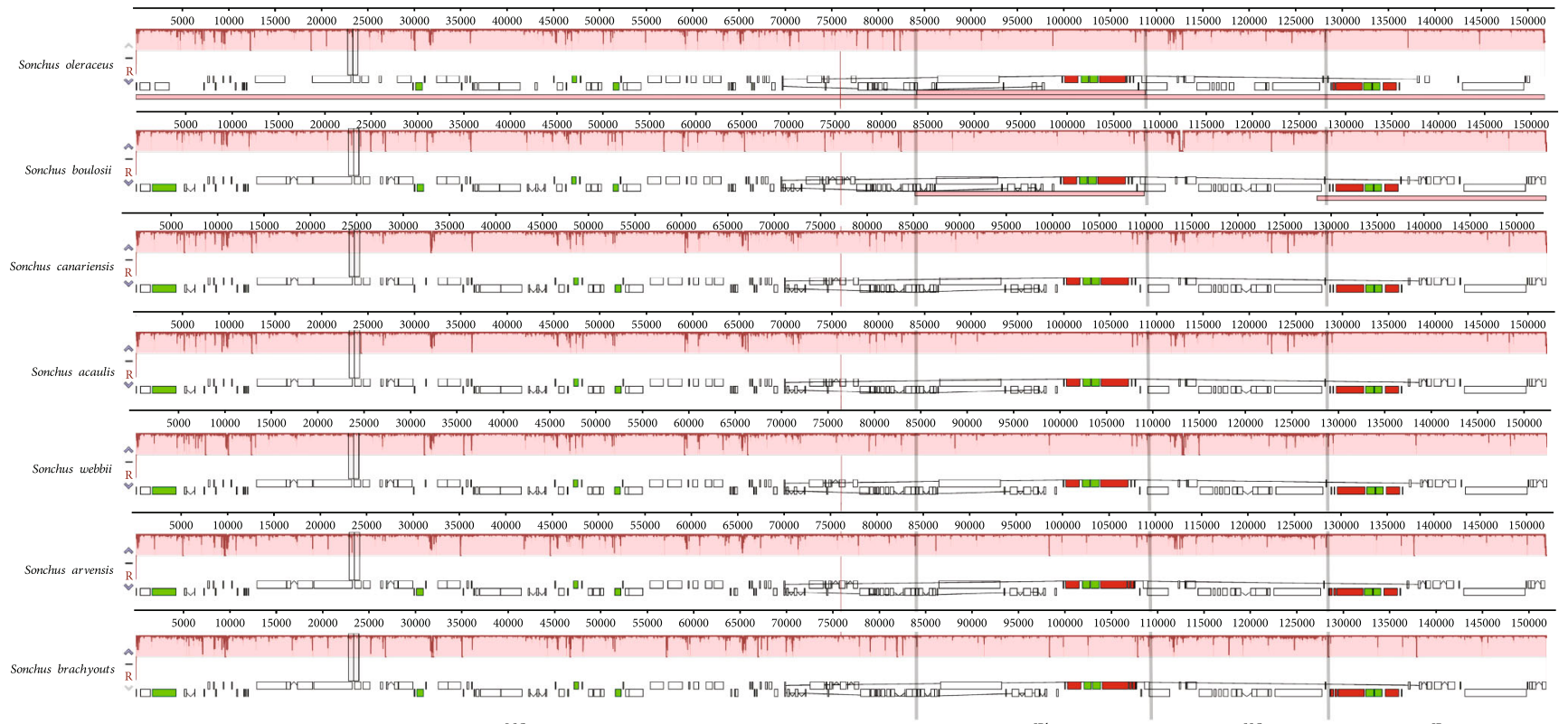

LSC

IRb

ssc

IRa

Figure 4: Mauve alignment of the 7 Sonchus chloroplast genomes. The rectangles represent the similarity between genomes, and the lines between rectangles represent a type of collinearity. The small square indicates the gene location in each genome. White represents CDSs, green represents tRNAs, and red represents rRNAs. 


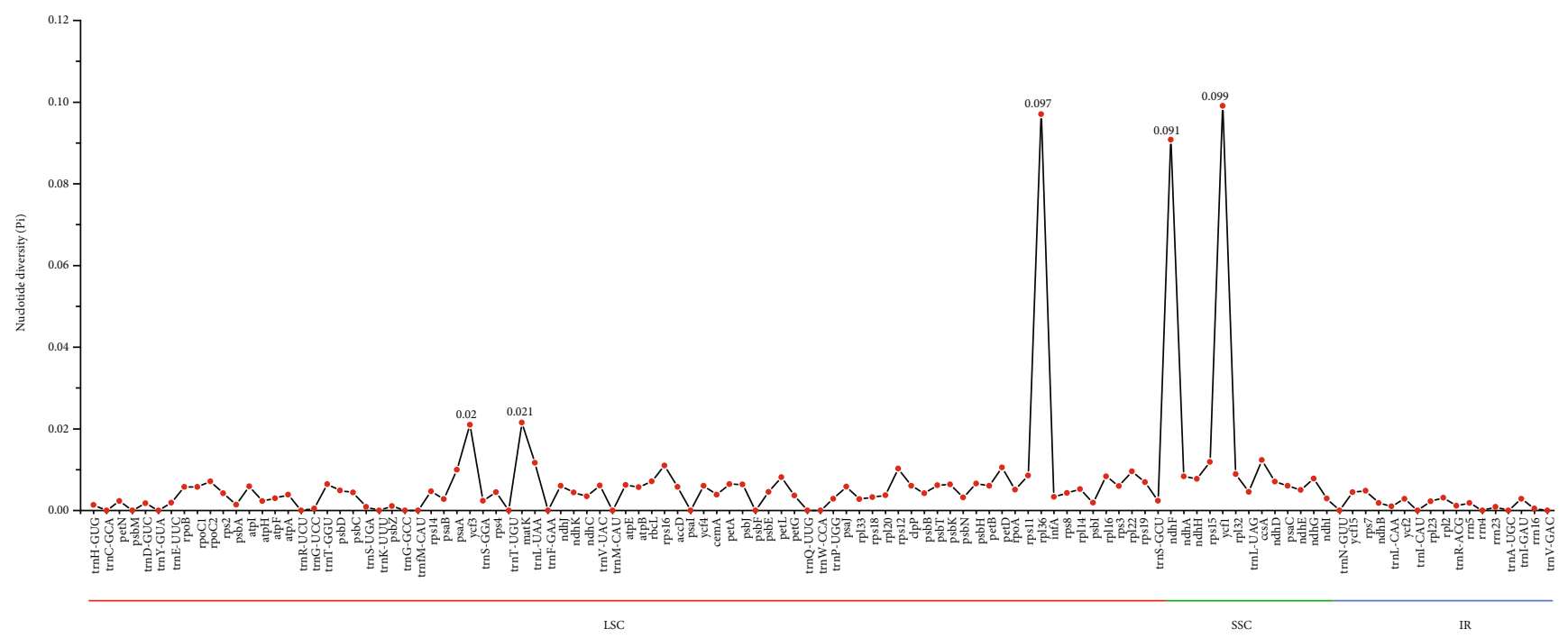

FIgURe 5: Nucleotide diversity (Pi) values among the 7 Sonchus species.

The number of variable sites in the IR region was more conserved than that in the LSC and SSC regions, and 5 of these sites were highly variable: $y c f 3$, matK, $r p l 36, n d h F$, and $y c f 1$. Three of the sites $(y c f 3$, matK, and $r p l 36)$ were located in the LSC region, and 2 ( $n d h F$ and $y c f 1$ ) were located in the SSC region (Figure 5). Five divergence hotspots in the most variable regions $(\mathrm{Pi}>0.02)$ could be used as potential molecular markers for phylogenetic studies of Sonchus species.

3.5. Phylogenetic Analysis. On the basis of the phylogenetic analysis of the cp genome relationships of 42 representative Compositae plants, the taxonomic status and evolutionary relationships of $S$. brachyotus were determined (Figure 6). The evolutionary tree revealed clear phylogenetic relationships for 43 species in 14 genera of Compositae, which were clustered into 3 branches. The first branch consists of 18 species in 4 genera, Lactuca, Mulgedium, Taraxacum, and Sonchus, all belonging to Lactuceae. The second branch consists of 11 species from 4 genera, Atractylodes, Cirsium, Carthamus, and Saussurea. The third branch consists of 14 species of 6 genera, Chrysanthemum, Artemisia, Leontopodium, Aster, Anaphalis, and Helianthus. Chrysanthemum and Artemisia belong to Anthemideae; Leontopodium and Anaphalis belong to Inuleae; Aster belongs to Astereae; and Helianthus belongs to the Heliantheae. These are all members of Cynareae. Sonchus is located on the first branch of the phylogenetic tree. In the Sonchus genus, S. brachyotus is more closely related to the small clades formed by $S$. arvensis and $S$. oleraceus, so it can be inferred that they have the closest relationship.

\section{Discussion}

As the second largest family in the plant kingdom, Compositae consists of approximately 1,620 genera and more than 23,600 species [36, 37]. Nevertheless, few cp genomic sequences for members of this family have been stored in GenBank, with the first sequence being that of $L$. sativa
[38, 39]. Although the advancement of high-throughput sequencing techniques has enabled several additional Compositae cp genomes to be sequenced [40-43], the cp genome of $S$. brachyotus has remained unexplored. In this study, we sequenced the complete cp genome of $S$. brachyotus by using Illumina high-throughput sequencing technology.

The structure and genes of the cp genome of S. brachyotus were found to be highly conserved through comparative analysis with closely related species, and they exhibited the same protein-coding genes, tRNAs, and rRNAs. Nevertheless, there was a difference in genome size (Table 1), indicating genetic differences. We found that this phenomenon may be due to contractions and expansions of boundary regions [44-48]. The length of the cp genome sequence is related to the contraction and expansion of noncoding regions. Recent studies have revealed that the IRb/SSC and IRa/LSC regions are mainly responsible for length differences in cp genome sequences, and such regions have been discovered in numerous angiosperm cp genome sequences [49]. Cho et al. [1, 50] carried out a boundary analysis of the LSC, SSC, and IR regions of the cp genomes of 5 Sonchus plants and found some slight differences in the position or length of the $r p s 19, \quad r p l 2, \operatorname{trn} H, n d h F$, and $y c f 1$ genes. Although the whole genome structure, including both gene number and order, was found to be nearly identical, the cp genome of $S$. brachyotus and the 6 published cp genomes of Sonchus (S. oleraceus, S. boulosii, S. canariensis, S. acaulis, $S$. webbii, and $S$. arvensis) showed obvious deviations at the $\mathrm{IRb} / \mathrm{SSC}$ and IRa/LSC borders.

Microsatellites can be divided into mono-, di-, tri-, tetra, penta-, and hexanucleotide repeats. The locations of SSRs have functional roles in the genome, including gene regulation, advancement, and evolution. As shown in a genomewide analysis of polymorphisms related to height, microsatellite markers can be powerful tools for measuring genetic diversity in populations and addressing genetic issues, such as gene origin, flow, and species group configuration, at the level of both intraspecific and interspecific variations [51]. 


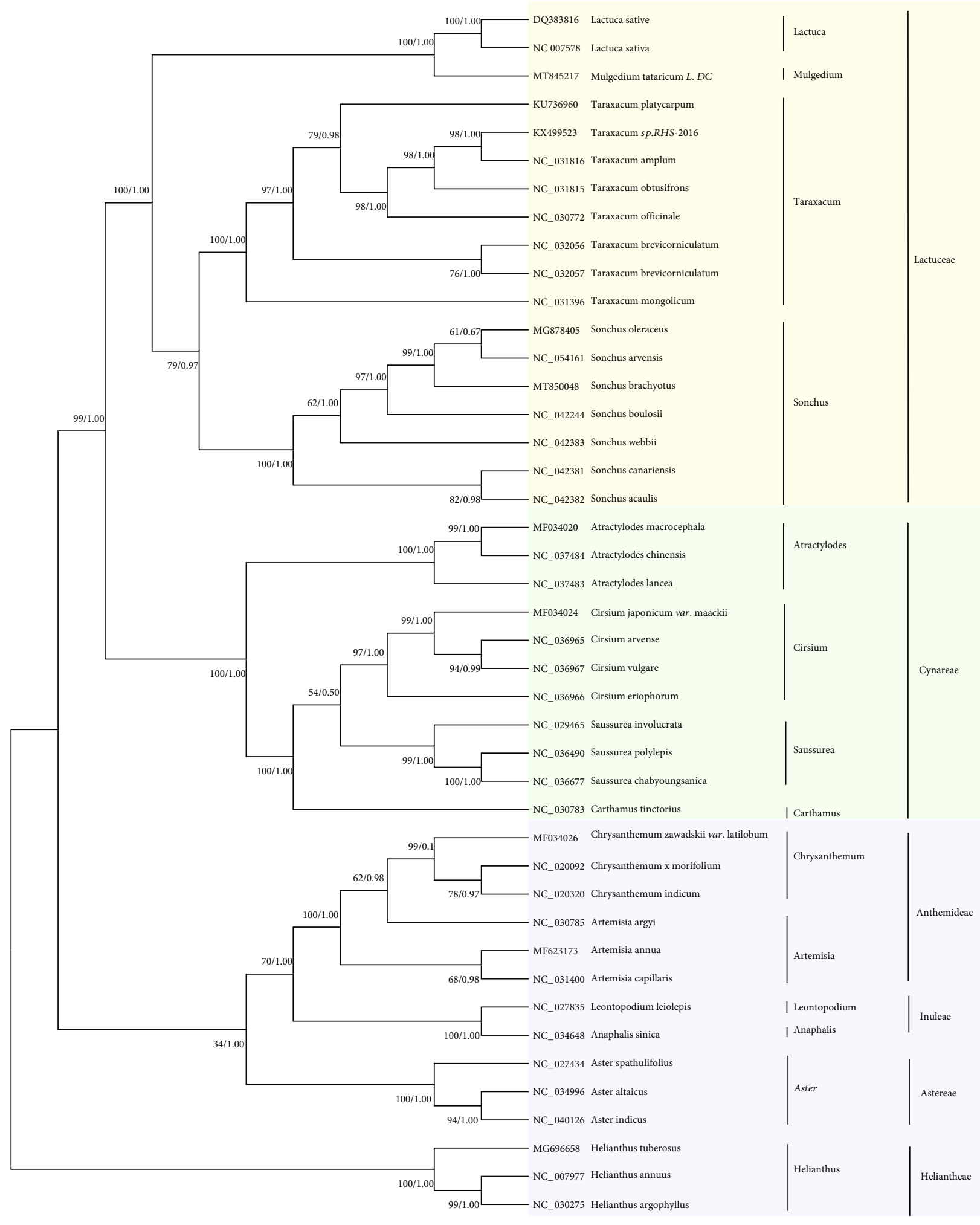

FIGURE 6: Phylogenetic analysis of the chloroplast genomes of 43 Compositae plants using maximum parsimony and Bayesian inference. MP bootstrap value/Bayesian posterior probability given at each node. The pentastar in the phylogenetic tree indicated that the support rate of the branch is 100/1.0. The yellow area is the first branch, the green area is the second branch, and the purple area is the third branch.

Population-specific cp SSR polymorphisms have also been documented in other plant species, such as Pinus sylvestris L. [52], Triticum spp. [53], Abies alba Mill. [54], and Cucu- mis spp. [55]. Repeat motifs play a crucial role in phylogenies, and they are valuable because of their applicability to genome rearrangement analysis [56]. Cho et al. [1, 50] 
analyzed the SSRs of the cp genomes of 5 species of Sonchus and found that the SSRs were mainly distributed in coding regions and LSC regions. In our study, 175 repeat sequences were found in S. brachyotus; additionally, we discovered that they mostly existed in the LSC regions.

Previous studies show that multiple sequence alignments used for interspecies discrimination can reveal the development of mutational hotspots [57, 58] and be applied in phylogenetic or phylogeographic studies [59, 60]. At present, some studies have shown that markers derived from chloroplast genomes can also be used in phylogenetic studies [61]. In several studies, the LSC and SSC regions were less conserved than the IR region [61-63], as revealed in this study. Numerous variable sites (e.g., $y c f 3$, matK, $r p l 36, n d h F$, and $y c f 1$ ) were confirmed by calculating and comparing the nucleotide diversity value (Pi). Among them, $y c f 1$ and $y c f 3$ have been demonstrated to be conducive markers for phylogenetic studies of Sonchus $[1,50]$. These markers were also found to be useful for analyzing the intraspecific variation of S. brachyotus. According to the results of the present study, 5 divergence hotspots screened on the basis of $\mathrm{Pi}>0.02$ show great potential for the development of a system of highly informative markers for S. brachyotus.

The taxonomic position and evolutionary relationships of $S$. brachyotus were revealed through comparisons with 42 Compositae plants, which were based on the correlations of all $\mathrm{cp}$ genomes. The 43 Compositae plants were divided into 3 groups. The phylogenetic relationships identified among Sonchus species were consistent with those from previous studies $[1,50,64]$. James et al. [64] constructed a phylogeny of 13 species of Compositae plants on the basis of the cp genome and revealed that $S$. oleraceus was closely related to L. sativa (AP007232). Cho et al. [1] used cp genomes to analyze a phylogeny of 32 Compositae plants and revealed that S. acaulis, S. canariensis, and $S$. webbii were closely related to $S$. oleraceus (MG 878405). Cho et al. [50] utilized cp genomes to analyze a phylogeny of 30 Compositae plants and demonstrated that $2 \mathrm{~S}$. asper and $2 \mathrm{~S}$. oleraceus plants were closely related to $S$. oleraceus (MG 878405). Overall, $S$. oleraceus was closely related to $S$. asper. In this study, Sonchus was most closely related to Taraxacum, followed by Lactuca. S. arvensis is the closest relative of S. brachyotus, followed by $S$. oleraceus, within the Sonchus genus. Therefore, we hypothesize that S. brachyotus and S. arvensis show similarity in physiology. Phylogenetic relationships identified within Sonchus and its phylogenetic relationships with other genera of the Compositae can facilitate additional studies. The $\mathrm{cp}$ genome sequences provide useful genetic information for understanding the evolution of Compositae plants.

\section{Conclusions}

In this study, we assembled, annotated, and analyzed the cp genome of $S$. brachyotus, an important wild plant used for food and medicine. The $S$. brachyotus $\mathrm{cp}$ genome $(151,977 \mathrm{bp})$ was fully characterized and compared with those of related species. We identified IR regions, as well as SSC and LSC regions. The S. brachyotus cp genome included 132 genes, of which there were 87 proteincoding genes, 8 rRNA genes, and 37 tRNA genes. A total of 175 microsatellites and 35 pairs of repeat sequences were detected in the cp genome of $S$. brachyotus. The unique inversion, insertion, and gene loss events detected here may provide informative markers for phylogenetic resolution among different genera in Compositae. Several hotspots (e.g., $y c f 3$, matK, rpl36, ndhF, and $y c f 1$ ) of intergeneric divergence were also identified. Both RAxML and GTR analyses strongly support the topology in which the clade including $S$. brachyotus is near that containing $S$. arvensis. The cp genomic resources presented in this study will be useful for further studies on the evolutionary patterns of $S$. brachyotus and its closely related species.

\section{Data Availability}

The data that support the findings of this study are openly available in GenBank of NCBI at https://www.ncbi.nlm.nih gov/, and the accession numbers are provided in Table S1 in Supplementary Materials.

\section{Disclosure}

The funding bodies had no role in the study design, analysis and interpretation of data, or writing of the manuscript.

\section{Conflicts of Interest}

The authors declare no competing interests.

\section{Authors' Contributions}

C.W. conceived this study. C.W. and J.L. performed the experiments and drafted the manuscript. M.L. contributed to material management and sample collection. Y.S. contributed to manuscript preparation. X.X. contributed to data interpretation. C.W. and J.S. helped revise the manuscript. All authors reviewed the manuscript. Caixiang Wang and Juanjuan Liu contributed equally to this work and should be considered co-first authors.

\section{Acknowledgments}

We thank Danxia He, Ph.D., from the College of Traditional Chinese Pharmacy, China Pharmaceutical University, for helping us identify the species Sonchus brachyotus DC. This work was supported by the Science and Technology Innovation Funds of Gansu Agricultural University (GAU-KYQD2018-32), National Natural Science Foundation of China (31801414), and Student Research Training Program of Gansu Agricultural University (202011032, 202011034).

\section{Supplementary Materials}

Table S1: list of chloroplast sequences included in the phylogenetic analyses. Figure S1: pictures of Sonchus brachyotus. (Supplementary Materials) 


\section{References}

[1] M. S. Cho, J. Y. Yang, T. J. Yang, and S. C. Kim, "Evolutionary comparison of the chloroplast genome in the woody Sonchus alliance (Asteraceae) on the Canary Islands," Genes (Basel), vol. 10, no. 3, p. 217, 2019.

[2] N. Kilian, B. Gemeinholzer, and H. W. Lack, "Tribe Cichorieae," in Systematics, Evolution, and Biogeography of Compositae, V. A. Funk, A. Susanna, T. Stuessy, and R. Bayer, Eds., pp. 343-383, International Association for Plant Taxonomy, Vienna, 2009.

[3] N. Kilian, R. Hand, and E. von Raab-Straube, Cichorieae Systematics Portalhttp://cichorieae.e-taxonomy.net/portal/.

[4] I. A. Al-Shehbaz, B. Bartholomew, D. E. Boufford et al., Flora of China, vol. 80, Science Press (Beijing), Beijing, 1997.

[5] V. C. O. M. Jiangsu, Dictionary of traditional Chinese medicine, Shanghai Scientific \& Technical Publishers, Shanghai, 1986.

[6] L. Liu, J. Li, and Y. Chen, "Analysis of fatty acids content in the stems and leaves of the Sonchus brachyotus DC., Sonchus oleraceus L. and lxeris denticulata Stebb," Journal of Jilin University (Medicine Edition), vol. 6, pp. 606-607, 2002.

[7] J. Li, G. Yang, L. Ma et al., "Study on hypoglycemic effect of water extract of Sonchus L. on experimental diabetic mice," Lishizhen Medicine and Materia Medica Research, vol. 22, no. 2, pp. 419-421, 2011.

[8] D. Z. Xia, X. F. Yu, Z. Y. Zhu, and Z. D. Zou, “Antioxidant and antibacterial activity of six edible wild plants (Sonchus spp.) in China," Natural Product Research, vol. 25, no. 20, pp. 18931901, 2011.

[9] Z. Xie and Y. Yu, Classification of Chinese Herbal Medicine, People's Medical Publishing House, Beijing, 1996.

[10] H. E. Yan-Yun, L. I. Xiao-ming, Z. H. Cai-yan, and H. U. Zi-yi, "Apoptosis of lung cancer line A549 induced by Sonchus branchyotus DC. Aqueous extracts," Natural Product Research and Development, vol. 26, no. 9, pp. 1380-1384, 2014.

[11] F. Pan, H. Y. Zhang, X. M. Li et al., "Effect of quality control on the total antioxidant capacity of the extract from Sonchus brachyotus DC," International Journal of Food Properties, vol. 21, no. 1, pp. 1362-1370, 2018.

[12] Q. I. E. Pei-juan, D. Xu-chang, W. Min, and L. I. Xiu-zhong, "Antioxidant activity of each polar composition from methanol extracts of Sonchus brachyotus DC," Science and Technology of Food Industry, vol. 37, no. 16, pp. 146-156, 2016.

[13] C. W. J. Birky, "Uniparental inheritance of mitochondrial and chloroplast genes: mechanisms and evolution," Proceedings of the National Academy of Sciences of the United States of America, vol. 92, no. 25, pp. 11331-11338, 1995.

[14] S. Asaf, A. L. Khan, A. R. Khan et al., "Complete chloroplast genome of Nicotiana otophora and its comparison with related species," Frontiers in Plant Science, vol. 7, p. 843, 2016.

[15] J. Carbonell-Caballero, R. Alonso, V. Ibañez, J. Terol, M. Talon, and J. Dopazo, "A phylogenetic analysis of 34 chloroplast genomes elucidates the relationships between wild and domestic species within the genus citrus," Molecular Biology and Evolution, vol. 32, no. 8, pp. 2015-2035, 2015.

[16] S. Hong, K. S. Cheon, K. O. Yoo, H. O. Lee, M. Mekapogu, and K. S. Cho, "Comparative analysis of the complete chloroplast genome sequences of three Amaranthus species," Plant Genetic Resources: Characterization and Utilization, vol. 17, no. 3, pp. 245-254, 2019.
[17] Q. Chen, X. Wu, and D. Zhang, "Phylogenetic analysis of Fritillaria cirrhosa D. Don and its closely related species based on complete chloroplast genomes," PeerJ, vol. 7, article e7480, 2019.

[18] Y. P. du, Y. Bi, F. P. Yang et al., "Complete chloroplast genome sequences of Lilium : insights into evolutionary dynamics and phylogenetic analyses," Scientific Reports, vol. 7, no. 1, p. 5751, 2017.

[19] Y. Hu, K. E. Woeste, and P. Zhao, "Completion of the chloroplast genomes of five Chinese Juglans and their contribution to chloroplast phylogeny," Frontiers in Plant Science, vol. 7, p. 1955, 2017.

[20] Y. Yang, T. Zhou, D. Duan, J. Yang, L. Feng, and G. Zhao, "Comparative analysis of the complete chloroplast genomes of five Quercus species," Frontiers in Plant Science, vol. 7, p. 959, 2016.

[21] W. Zhai, X. Duan, R. Zhang et al., "Chloroplast genomic data provide new and robust insights into the phylogeny and evolution of the Ranunculaceae," Molecular Phylogenetics and Evolution, vol. 135, pp. 12-21, 2019.

[22] J. J. Doyle, "A rapid DNA isolation procedure for small quantities of fresh leaf tissue," Phytochemical Bulletin, vol. 19, no. 1, pp. 11-15, 1987.

[23] R. K. Patel and M. Jain, "NGS QC toolkit: a toolkit for quality control of next generation sequencing data," PLoS One, vol. 7, no. 2, article e30619, 2012.

[24] H. Li and R. Durbin, "Fast and accurate short read alignment with burrows-wheeler transform," Bioinformatics, vol. 25, no. 14, pp. 1754-1760, 2009.

[25] A. Bankevich, S. Nurk, D. Antipov et al., "SPAdes: a new genome assembly algorithm and its applications to single-cell sequencing," Journal of Computational Biology, vol. 19, no. 5, pp. 455-477, 2012.

[26] S. Kurtz, A. Phillippy, A. L. Delcher et al., "Versatile and open software for comparing large genomes," Genome Biology, vol. 5, no. 2, p. R12, 2004.

[27] M. Lohse, O. Drechsel, S. Kahlau, and R. Bock, “OrganellarGenomeDRAW-a suite of tools for generating physical maps of plastid and mitochondrial genomes and visualizing expression data sets," Nucleic Acids Research, vol. 41, no. W1, pp. W575-W581, 2013.

[28] A. Amiryousefi, J. Hyvonen, and P. Poczai, "IRscope: an online program to visualize the junction sites of chloroplast genomes," Bioinformatics, vol. 34, no. 17, pp. 3030-3031, 2018.

[29] A. E. Darling, B. Mau, and N. T. Perna, "progressiveMauve: multiple genome alignment with gene gain, loss and rearrangement," PLoS One, vol. 5, no. 6, article e11147, 2010.

[30] K. Katoh and D. M. Standley, "MAFFT multiple sequence alignment software version 7: improvements in performance and usability," Molecular Biology and Evolution, vol. 30, no. 4, pp. 772-780, 2013.

[31] P. Librado and J. Rozas, "DnaSP v5: a software for comprehensive analysis of DNA polymorphism data," Bioinformatics (Oxford, England), vol. 25, no. 11, pp. 1451-1452, 2009.

[32] K. Katoh and D. M. Standley, "A novel method for rapid multiple sequence alignment based on fast Fourier transform," Nucleic Acids Research, vol. 30, no. 14, pp. 30593066, 2002.

[33] K. Tamura, G. Stecher, and S. Kumar, "MEGA11: molecular evolutionary genetics analysis version 11," Molecular Biology and Evolution, vol. 38, no. 7, pp. 3022-3027, 2021. 
[34] F. Ronquist, M. Teslenko, P. van der Mark et al., "MrBayes 3.2: efficient Bayesian phylogenetic inference and model choice across a large model space," Systematic Biology, vol. 61, no. 3, pp. 539-542, 2012.

[35] D. Posada and K. A. Crandall, "MODELTEST: testing the model of DNA substitution," Bioinformatics, vol. 14, no. 9, pp. 817-818, 1998.

[36] J. L. Panero and B. S. Crozier, “Asteraceae. Sunflowers, daisies," The Tree of Life Web Project, 2012.

[37] P. F. Stevens, “Angiosperm Phylogeny Website," 2001, http:// www.mobot.org/MOBOT/research/APweb/.

[38] K. Shinozaki, M. Ohme, M. Tanaka et al., "The complete nucleotide sequence of the tobacco chloroplast genome: its gene organization and expression," The EMBO Journal, vol. 5, no. 9, pp. 2043-2049, 1986.

[39] Y. Somaratne, D. L. Guan, W. Q. Wang, L. Zhao, and S. Q. Xu, "Complete chloroplast genome sequence of Xanthium sibiricum provides useful DNA barcodes for future species identification and phylogeny," Plant Systematics and Evolution, vol. 305, no. 10, pp. 949-960, 2019.

[40] Y. Zhang, L. Li, T. L. Yan, and Q. Liu, “Complete chloroplast genome sequences of Praxelis (Eupatorium catarium Veldkamp), an important invasive species," Gene, vol. 549, no. 1, pp. 58-69, 2014.

[41] P. L. Curci, D. de Paola, D. Danzi, G. G. Vendramin, and G. Sonnante, "Complete chloroplast genome of the multifunctional crop globe artichoke and comparison with other Asteraceae," PLoS One, vol. 10, no. 3, article e0120589, 2015.

[42] M. S. RH, L. Majeský, T. Schwarzacher, R. Gornall, and P. Heslop-Harrison, "Complete chloroplast genomes from apomictic Taraxacum (Asteraceae): identity and variation between three microspecies," PLoS One, vol. 12, no. 2, article e0168008, 2017.

[43] X. Shen, M. Wu, B. Liao et al., "Complete chloroplast genome sequence and phylogenetic analysis of the medicinal plant Artemisia annua," Molecules, vol. 22, no. 8, p. 1330, 2017.

[44] L. Wang, N. He, Y. Li, Y. Fang, and F. Zhang, "Complete chloroplast genome sequence of Chinese lacquer tree (Toxicodendron vernicifluum, Anacardiaceae) and its phylogenetic significance," BioMed Research International, vol. 2020, Article ID 9014873, 13 pages, 2020.

[45] R. Kolodner, K. K. Tewari, and R. C. Warner, "Physical studies on the size and structure of the covalently closed circular chloroplast DNA from higher plants," Biochimica et Biophysica Acta, vol. 447, no. 2, pp. 144-155, 1976.

[46] Y. Song, W. Dong, B. Liu et al., "Comparative analysis of complete chloroplast genome sequences of two tropical trees Machilus yunnanensis and Machilus balansae in the family Lauraceae," Frontiers in Plant Science, vol. 6, p. 662, 2015.

[47] K. Kim and H. Lee, "Complete chloroplast genome sequences from Korean ginseng (Panax schinseng Nees) and comparative analysis of sequence evolution among 17 vascular plants," DNA research: an international journal for rapid publication of reports on genes and genomes, vol. 11, no. 4, pp. 247-261, 2004.

[48] L. A. Raubeson, R. Peery, T. W. Chumley et al., "Comparative chloroplast genomics: analyses including new sequences from the angiosperms Nuphar advena and Ranunculus macranthus," BioMed Central genomics, vol. 8, p. 174, 2007.
[49] R. Lu, P. Li, and Y. Qiu, "The complete chloroplast genomes of three Cardiocrinum (Liliaceae) species: comparative genomic and phylogenetic analyses," Frontiers in Plant Science, vol. 7, p. 2054, 2017.

[50] M. Cho, J. H. Kim, C. S. Kim, J. A. Mejías, and S. C. Kim, “Sow thistle chloroplast genomes: insights into the plastome evolution and relationship of two weedy species, Sonchus asper and Sonchus oleraceus (Asteraceae)," Genes, vol. 10, no. 11, p. 881, 2019.

[51] M. L. Wang, N. A. Barkley, and T. M. Jenkins, "Genomes and genomics @2009 global science books microsatellite markers in plants and insects. Part I: applications of biotechnology," Genes Genomes, vol. 3, no. 1, 2009.

[52] J. Provan, N. Soranzo, N. J. Wilson et al., "Gene-pool variation in Caledonian and European Scots pine (Pinus sylvestris L.) revealed by chloroplast simple-sequence repeats," Proceedings. Biological sciences, vol. 265, no. 1407, pp. 1697-1705, 1998.

[53] T. Ishii, N. Mori, and Y. Ogihara, "Evaluation of allelic diversity at chloroplast microsatellite loci among common wheat and its ancestral species," Theoretical and Applied Genetics, vol. 103, no. 6-7, pp. 896-904, 2001.

[54] G. G. Vendramin, B. Degen, R. J. Petit, M. Anzidei, A. Madaghiele, and B. Ziegenhagen, "High level of variation at Abies alba chloroplast microsatellite loci in Europe," Molecular Ecology, vol. 8, no. 7, pp. 1117-1126, 1999.

[55] S. Chung, J. E. Staub, and J. Chen, "Molecular phylogeny of Cucumis species as revealed by consensus chloroplast SSR marker length and sequence variation," Genome, vol. 49, no. 3, pp. 219-229, 2006.

[56] T. Cavalier-Smith, "Chloroplast evolution: secondary symbiogenesis and multiple losses," Current Biology, vol. 12, no. 2, pp. R62-R64, 2002.

[57] I. Ahmed, P. J. Matthews, P. J. Biggs, M. Naeem, P. A. McLenachan, and P. J. Lockhart, "Identification of chloroplast genome loci suitable for high-resolution phylogeographic studies of Colocasia esculenta (L.) Schott (Araceae) and closely related taxa," Molecular Ecology Resources, vol. 13, no. 5, pp. 929-937, 2013.

[58] Z. Yang, T. Zhao, Q. Ma, L. Liang, and G. Wang, "Comparative genomics and phylogenetic analysis revealed the chloroplast genome variation and interspecific relationships of Corylus (Betulaceae) species," Frontiers in Plant Science, vol. 9, p. 927, 2018.

[59] J. Shaw, H. L. Shafer, O. R. Leonard, M. J. Kovach, M. Schorr, and A. B. Morris, "Chloroplast DNA sequence utility for the lowest phylogenetic and phylogeographic inferences in angiosperms: the tortoise and the hare IV," American Journal of Botany, vol. 101, no. 11, pp. 1987-2004, 2014.

[60] S. R. Downie and R. K. Jansen, "A comparative analysis of whole plastid genomes from the Apiales: expansion and contraction of the inverted repeat, mitochondrial to plastid transfer of DNA, and identification of highly divergent noncoding regions," Systematic Botany, vol. 40, no. 1, pp. 336351, 2015.

[61] V. C. Biju, S. Pr, S. Vijayan et al., "The complete chloroplast genome of Trichopus zeylanicus, and phylogenetic analysis with Dioscoreales," Plant Genome, vol. 12, no. 3, pp. 1-11, 2019.

[62] Z. Zhao, X. Wang, Y. Yu et al., "Complete chloroplast genome sequences ofDioscorea: characterization, genomic resources, and phylogenetic analyses," PeerJ, vol. 6, article e6032, 2018. 
[63] L. Ma, P. Ma, and D. Li, "The first complete plastid genome of _Burmannia disticha_ L. from the mycoheterotrophic monocot family Burmanniaceae," Plant Divers, vol. 40, no. 5, pp. 232-237, 2018.

[64] P. H. James, A. W. Jeff, F. T. David, M. Keenan, B. S. Chauhan, and G. H. Walter, "Complete chloroplast genome of glyphosate resistant Sonchus oleraceus L. from Australia, with notes on the small single copy (SSC) region orientation," Mitochondrial DNA Part B, vol. 3, no. 1, pp. 363-364, 2018. 